\title{
Detection of Nucleic Acid-Nuclear Hormone Receptor Complexes with Mass Spectrometry
}

\author{
Claudia Bich, ${ }^{\mathrm{a}}$ Cédric Bovet, ${ }^{\mathrm{a}}$ Natacha Rochel, ${ }^{\mathrm{b}}$ Carole Peluso-Iltis, ${ }^{\mathrm{b}}$ \\ Andreas Panagiotidis, ${ }^{\mathrm{c}}$ Alexis Nazabal, ${ }^{\mathrm{d}}$ Dino Moras, ${ }^{\mathrm{b}}$ and \\ Renato Zenobi ${ }^{\mathrm{a}}$ \\ a Department of Chemistry and Applied Biosciences, ETH Zürich, Zürich, Switzerland \\ ${ }^{b}$ Institut de Génétique et de Biologie Moléculaire et Cellulaire CNRS, Illkirch, France \\ ${ }^{\mathrm{c}}$ Institute of Molecular Systems Biology (IMSB), ETH Zürich, Zürich, Switzerland \\ ${ }^{\mathrm{d}}$ CovalX AG, Schlieren, Switzerland
}

Nuclear receptors, such as the retinoic acid receptor (RAR) or the 9-cis retinoic acid receptor (RXR), interact not only with their ligands but also with other types of receptors and with DNA. Here, two complementary mass spectrometry (MS) methods were used to study the interactions between retinoic receptors (RXR/RAR) and DNA: non-denaturing nano-electrospray (nanoESI MS), and high-mass matrix-assisted laser desorption ionization (MALDI MS) combined with chemical cross-linking. The RAR-RXR heterodimer was studied in the presence of a specific DNA sequence (DR5), and a specific RAR.RXR.DNA complex was detected with both MS techniques. RAR by itself showed no significant homodimerization. A complex between RAR and the double stranded DR5 was detected with nanoESI. After cross-linking, high-mass MALDI mass spectra showed that the RAR binds the single stranded DR5, and the RAR dimer binds both single and double stranded DR5. Moreover, the MALDI mass spectrum shows a larger RAR dimer signal in the presence of DNA. These results suggest that a gene-regulatory site on DNA can induce quaternary structural changes in a transcription factor such as RAR. (J Am Soc Mass Spectrom 2010, 21, 635-645) (c) 2010 American Society for Mass Spectrometry

$\mathrm{R}$ etinoids (metabolites of vitamin A) exert a wide variety of effects on embryonic development, tissue homeostasis, cell proliferation, differentiation, and apoptosis [1]. Their pleiotropic effects are mediated through two transcriptional regulators, the retinoic acid nuclear receptors $(\operatorname{RAR} \alpha, \beta$, and $\gamma$ isotypes), and the retinoid X nuclear receptors $(\operatorname{RXR} \alpha, \beta$, and $\gamma$ isotypes), which form functional RAR/RXR heterodimer. RXR also forms a heterodimer with other nuclear receptors (NRs) [2,3] and, in certain cases, can act on its own or in concert with other signaling pathways to induce cell differentiation or apoptosis [4]. While RARs are activated by all-trans retinoic acid (atRA) and 9-cis retinoic acid (RA), RXRs are only activated by 9-cisRA, although the physiological RXR ligand remains unknown [5]. Retinoids and rexinoids (RXR selective) ligands are important drugs for cancer therapy and prevention, and for metabolic diseases [6].

NRs share a common modular organization with a variable amino-terminal domain, a conserved DNAbinding domain (DBD) and the ligand binding domain (LBD) that represses or activates gene transcription upon ligand binding and/or cofactor positioning. The

Address reprint requests to Professor R. Zenobi, Department of Chemistry and Applied Biosciences, ETH Zürich, CH-8093 Zürich, Switzerland.E-mail zenobi@org.chem.ethz.ch
LBD harbors numerous interaction surfaces for multiple protein partners, including corepressors, coactivators, and other bridging factors that participate in signal transduction towards the basal transcriptional machinery. Physiologically, most NRs function as dimers, resulting in the binding of two DBDs to neighboring hexanucleotide DNA motifs called hormone response elements (HREs). Heterodimeric NRs bind to asymmetric response elements with specific polarities that result from the formation of cooperative interfaces between DBDs.

All RXR heterodimers preferentially bind DNA on two sites of a direct repeat (DR) configuration, separated by 1 to 5 nucleic acids, called DR1 to DR5, respectively $[3,7,8]$. It has also been observed that these receptors have higher affinity and specificity for the DNA as dimers compared with the monomeric form [7]. Both the binding strength and the binding selectivity are determined by the relative orientation, spacing, and nucleotide sequence of the repeat motifs on DNA [9].

The structure and formation of the RAR.RXR heterodimer $[3,10,11]$ as well as the homodimer RXR, [3, 12] interacting with DR1 after RA activation have been previously studied. The structure of this DR1-RAR.RXR system is well known, however the structure the heterodimer with DR5 is still unknown [13]. RXR can bind to DR1 both as a homodimer and as a heterodimer with 
RAR. As the two direct repeats are separated by only one base, the two partners are positioned on both sides of the dsDNA (Figure 1). It has been established that on DR5, RXR occupies the $5^{\prime}$ half-site of the direct repeat of the double stranded DR5, while RAR occupies the $3^{\prime}$ half-site (Figure 1) [3,11]. Consequently, they will be located on the same side of the double strand (Figure 1). One interesting point, recently observed, is that a biological response induced by RA activation is present even in the absence of RXR, which suggests that RAR could also form a homodimer and interact with DNA [14]. It has been shown that RXR forms a heterodimer with various other receptors, but the potential dimerization of all-trans retinoic acid receptors induced by interaction with a gene-regulatory site has never been demonstrated.

Here we use a fast and sensitive analytical tool, mass spectrometry (MS), to determine the interactions between nuclear receptors and DNA. Since the introduction of soft ionization methods, MS has become a valuable technique for characterizing noncovalent complexes. Usually, electrospray ionization (ESI) [15] or nanoelectrospray (nanoESI) mass spectrometry is preferred over matrix-assisted laser desorption/ionization mass spectrometry (MALDI MS) [16, 17] for studying noncovalent complexes, such as protein-protein, proteinligand, protein-DNA, or DNA-DNA complexes. This is mainly because ESI is able to spray "native-like" aqueous solutions [18, 19]. However, special sample preparation procedures such as desalting, and the use of aqueous volatile solutions at $\mathrm{pH} \approx 7$ such as ammonium acetate, are necessary to keep noncovalent complexes intact. Desalting and related purification steps are not always necessary in the case of MALDI MS due to its higher salt tolerance. Although noncovalent complexes are easily disrupted in the MALDI process, either during sample preparation (mixture with acidic organic matrix) or during desorption/ionization, some studies have demonstrated the possibility of detecting noncovalent protein complexes by MALDI, using specialized protocols such as desorption/ionization by an IR laser
[20-22], using the first shot analysis [23], or by employing non-acidic matrices [24].

Recently, the application of MALDI MS has been extended to the area of large heteromeric noncovalent complexes by combining chemical cross-linking and high-mass detection [25-28]. Indeed, an effective strategy to prevent complex dissociation during the MALDI process is stabilization by chemical cross-linking [29]. Chemical cross-linking is well established and extensively used with proteins, especially for studying interactions, structural effect, or for purification such as immunoprecipitation [26, 30-33]. Concerning the crosslinking between proteins and DNA, the efficiency of UV cross-linking by laser irradiation by the activation of artificially introduced moieties such as azido groups has been shown [34-36]. In this fashion, DNA-protein complexes could be detected and studied by MS [34]. The UV cross-linking mechanism is still not fully understood [34, 36].

Here, native ESI and high-mass MALDI MS were used to study the interaction of nuclear receptors with a specific DNA sequence. ESI, for obtaining accurate masses and high-mass MALDI, can be combined to obtain complementary information on noncovalent complexes. First, we studied the interaction of the RAR.RXR heterodimer with DR5. Then, the nuclear receptor RAR alone was incubated with DNA and studied by both MS techniques. We also investigated the influence of the DNA on RAR dimerization.

\section{Experimental}

\section{Protein Expression and Purification}

Expression of mRXR $\triangle \mathrm{AB}$ (133-467), mRAR $\triangle \mathrm{AB}(84-$ 462): for simplification, this will, in the following, be referred to as RXR and RAR. The nuclear receptors were expressed in E. coli BL21 (DE3) cells harboring a pET15b plasmid encoding a N-ter hexahistidine tagged fusion protein for RAR and a pET31 plasmid encoding a non-tagged protein for RXR. Cells were grown in LB

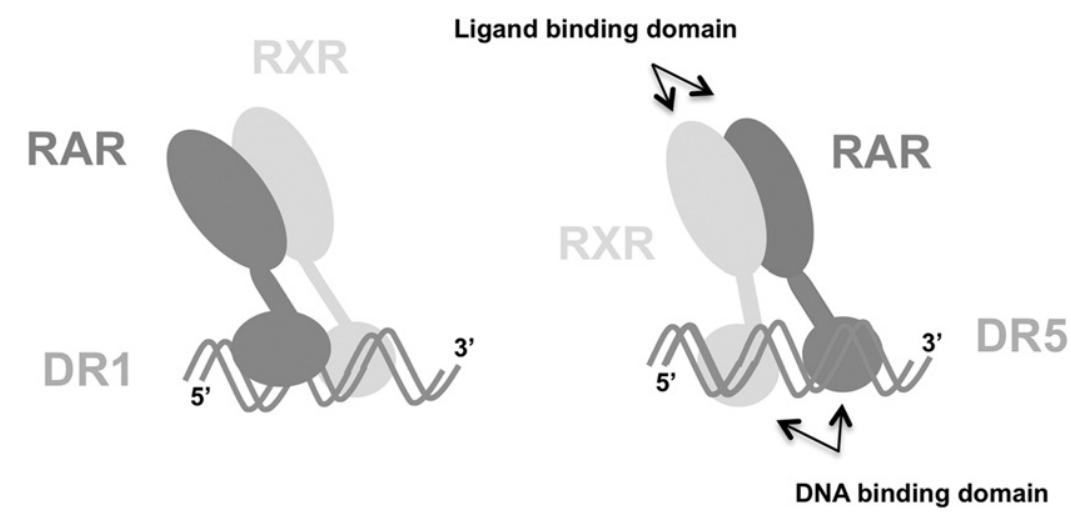

Figure 1. Schematic representation of the interaction between RAR, RXR, and DR1 (left) and the interaction between the heterodimer and DR5 (right). The ligand and DNA binding domains, schematically represented by a big and a small lobe, respectively, are sketched. The polarity of the DNA is only specified for the single strand containing the direct repeat. 
medium at $37^{\circ} \mathrm{C}$ until the absorbance at $600 \mathrm{~nm}$ reached 0.6-0.8. They were then allowed to grow for another $3 \mathrm{~h}$ at $25^{\circ} \mathrm{C}$ after induction with $0.8 \mathrm{mM}$ IPTG. Cells were harvested by centrifugation and stored at $-80{ }^{\circ} \mathrm{C}$ until further processing.

Purification: Cell pellets from 2L of His-RAR and from $2 \mathrm{~L}$ of His-RAR plus $4 \mathrm{~L}$ of RXR, respectively, were mixed and purified first by metal chelating affinity chromatography (nickel HiTrap crude FF; Pharmacia, Saint Quentin en Yvelines, France) with elution by imidazole steps. Protein fractions were then chromatographed on a Superdex S200 26/60 gel-filtration (Pharmacia) in Tris buffer (20 mM, pH7.5) containing 100 $\mathrm{mM} \mathrm{NaCl}, 100 \mathrm{mM} \mathrm{KCl}$, 5\% glycerol, $2 \mathrm{mM}$ Chaps, and $4 \mathrm{mM} \mathrm{MgSO}_{4}$. The ligand, 9-cis retinoic acid (RA) was then added as a saturated ethanol solution.

One percent of the total sample volume in a 4-fold molar excess, to saturate the receptors. This did not cause any detectable aggregation and was enough to saturate all receptor sites. All subsequent steps were performed under dimmed light to avoid photolysis or isomerization of the photosensitive ligand. In the crystal structure of RAR bound to 9-cis RA, measured in similar conditions, no isomerization was observed [37]. 2 mM Tris[2-carboxyethyl] phosphine (TCEP) was added and the proteins were then concentrated on Centricon30 (Centricon/Millipore, Molsheim, France) biological membranes. Purified single-stranded oligonucleotides from Eurogentec (Angers, France) were annealed in equimolar amounts at $85^{\circ} \mathrm{C}$ for $10 \mathrm{~min}$, and left to cool at $4{ }^{\circ} \mathrm{C}$. The sequence for the DR5 used in this study is the following: 5'-AGAGGTCAGACAGAGGTCAGA-3' /3' -TCTCCAGTCTGTTCCAGTCT-5', MW = 12,852 Da. The double stranded DNA DR5 contained the typical binding motif, in bold, (A/G)G(G/T)TCA separated by five nucleic acids [3]. The purified heterodimer RAR.RXR was mixed with a $1.1 \times$ molar excess of DR5 oligonucleotides and dialyzed overnight at $4{ }^{\circ} \mathrm{C}$ against Tris buffer $(10 \mathrm{mM}, \mathrm{pH} 7.5)$ containing 50 $\mathrm{mM} \mathrm{NaCl}, 50 \mathrm{mM} \mathrm{KCl}$, 5\% glycerol, $2 \mathrm{mM}$ Chaps, and $4 \mathrm{mM} \mathrm{MgSO}$. The heterodimeric DNA complex was further purified by gel-filtration on a Superdex S200 (Superdex/GE Healthcare, Lyon, France) 26/60 stationary phase. Fractions containing the RAR.RXR.DR5 complex were pooled and analyzed by native sodium dodecyl sulfate polyacrylamide gel electrophoresis (SDS-PAGE). As the His-tag was not detected during mass spectrometry measurements, we assume it gets lost during the purification.

\section{Materials}

Disuccinimidyl tartrate, iodoacetic acid N-hydroxysuccinimidyl ester, dithiobis (succinimidyl) propionate, and octanedioic acid di-N-hydroxysuccinimidyl ester (K100 MALDI MS analysis kit) were obtained from CovalX AG (Zurich, Switzerland). These cross-linkers are optimized for reacting in water or PBS buffer at $\mathrm{pH}$ 7-9. Sinapinic acid (SA) also came with the CovalX analysis kit. Single stranded DNA $\mathrm{C}_{3}, \mathrm{~A}_{9}$, and $\mathrm{C}_{9}$ were purchased from Microsynth AG (Balgach, Switzerland).

\section{Sample Preparation}

In all experiments, the ligand RA was always present in solution with the receptors. Before nanoESI MS measurements, the RAR, RAR.RXR, and DR5 stock solutions were desalted and buffer exchanged against 50 mM NH${ }_{4} \mathrm{OAc}$ ( $\mathrm{pH} 7.4$ ), using Micro Bio-Spin 6 columns (Bio-Rad, Reinach, Switzerland). DR5 was incubated with RAR for $15 \mathrm{~min}$ at room temperature before measurement. Relatively high concentrations (15-20 $\mu \mathrm{M})$ of each protein and DNA were used to obtain good spectra.

The RAR and the RAR.RXR stock solutions were diluted to $5.5 \mu \mathrm{M}$ and $5 \mu \mathrm{M}$, respectively, before MALDI measurements. In the case of DR5 as well as non-specific $\mathrm{A}_{9}$ and $\mathrm{C}_{9}$, a $5 \mu \mathrm{M}$ solution was incubated with the proteins $15 \mathrm{~min}$ before adding the cross-linker. The cross-linking reactions were carried out using a solution containing the abovementioned cocktail of cross-linkers specific for amino groups (K100). The protein complexes, with or without DNA, were stabilized with the cross-linking mixture at about 50-fold molar excess, freshly made, in a total volume of $10 \mu \mathrm{L}$. The sample and the cross-linker were incubated for 120 min at room temperature to allow the reaction to go to completion. As RA ligand is light sensitive, tubes were covered with aluminium foil during incubation. After cross-linking, $1 \mu \mathrm{L}$ of the sample containing the protein complex was directly mixed with $1 \mu \mathrm{L}$ of matrix solution. The freshly made matrix solution contained SA $(10 \mathrm{mg} / \mathrm{mL})$ in acetonitrile:water $(1: 1, \mathrm{vol} / \mathrm{vol})$ acidified with $0.1 \%$ (vol/vol) trifluoroacetic acid (TFA). After mixing, $1 \mu \mathrm{L}$ of the sample was deposited on the sample plate for MALDI MS analysis.

\section{Mass Spectrometry}

ESI mass spectra were acquired on a quadrupole timeof-flight mass spectrometer (Q-TOF ULTIMA; Waters, Manchester, UK) equipped with an automated chipbased nanoESI system (Nanomate 100, Advion Biosciences, Ithaca, NY, USA). To prevent dissociation of ligand-bound RAR, the source pressure of the mass spectrometer was raised to 3 mbar with a Speedivalve (BOC Edwards, La Chaux-de-Fonds, Switzerland). Under the conditions of high-pressure, the ion collisions with the residual gas are less energetic, thus leaving the noncovalent complexes intact. The transfer voltages of the mass spectrometer were tuned so as to preserve the noncovalent complexes during their transfer from the solution phase to the mass spectrometer vacuum, as previously described [38]. Best spectra obtained using optimum conditions are shown here; however these conditions were not the same for each case. Standard Q-TOF instruments have a quadrupole with limited ion transmission [39]. Using our Q-TOF instrument, it is 
possible to work up to $m / z 7000$ with optimization of parameters and if one is willing to tolerate poor resolution. In principle, quadrupoles on these instruments can be upgraded for optimal transmission of ions up to $\mathrm{m} / \mathrm{z} 32,000$ [40], but unfortunately this technology was not available in our lab.

MALDI mass spectra were acquired on a time-offlight mass spectrometer (Reflex IV; Bruker Daltonics $\mathrm{GmbH}$, Bremen, Germany) equipped with a high-mass detection system (HM1; CovalX AG, Zurich, Switzerland), which is a modified, commercial version of a design previously described by Hillenkamp and coworkers [16, 41]. The high-mass detection system provides high sensitivity with low saturation in the high mass range up to $>1 \mathrm{MDa}$. Typical accelerating voltages were $20 \mathrm{kV}$; the gain voltage on the detector was set to $2.8 \mathrm{kV}$, and the delayed extraction time was automatically adapted by the instrument's software according to the selected mass range. Spectra were averaged over 300 laser shots at different locations of the sample.

\section{Data Processing}

Before data processing, each MALDI and nanoESI mass spectrum was background-subtracted and smoothed with Complex Tracker 1.1 (CovalX AG) or the MassLynx 4.0 software (Waters), respectively. Complex Tracker permits to overlay control and cross-linked spectra and also to subtract them to clearly detect the peaks corresponding to the complexes formed.

\section{Results and Discussion}

\section{Mass Spectrometric Detection of RAR.RXR and $R A R \cdot R X R \cdot D N A$ Complexes}

The two receptors, RAR and RXR, were incubated with 9-cis RA ligand as described in the experimental part. The mixture of ligand-bound RAR, RXR and DR5 was analyzed with nanoESI MS. The nanoESI mass spectrum shows a major ion distribution between $\mathrm{m} / \mathrm{z} 4700$ and 6000, corresponding to a molecular weight of 94.77 $\mathrm{kDa}$ (Figure 2), in agreement with the theoretical molecular weight of a 2RA·RAR·RXR·dsDNA complex $(\mathrm{MW}=94,558)$. The mass difference is due to some unspecific clustering with solvent/buffer molecules, a fact that also leads to peak broadening. Harsher conditions did not allow the detection of the complex anymore; it was not possible to obtain sharper peaks. The ion distribution corresponding to the free nuclear receptor RXR $(37,390 \mathrm{Da})$ can also be observed in the spectrum. The peaks between $\mathrm{m} / \mathrm{z} 3500$ and 4500 are attributed to RXR, RAR, and parts of the 2RA.RAR. RXR.dsDNA complex charge state distribution but, for simplicity, are not labeled in the spectrum. The presence of some free RXR and RAR suggests that the complex is in equilibrium with its components in solution. Some dissociation may also result from the ioniza-

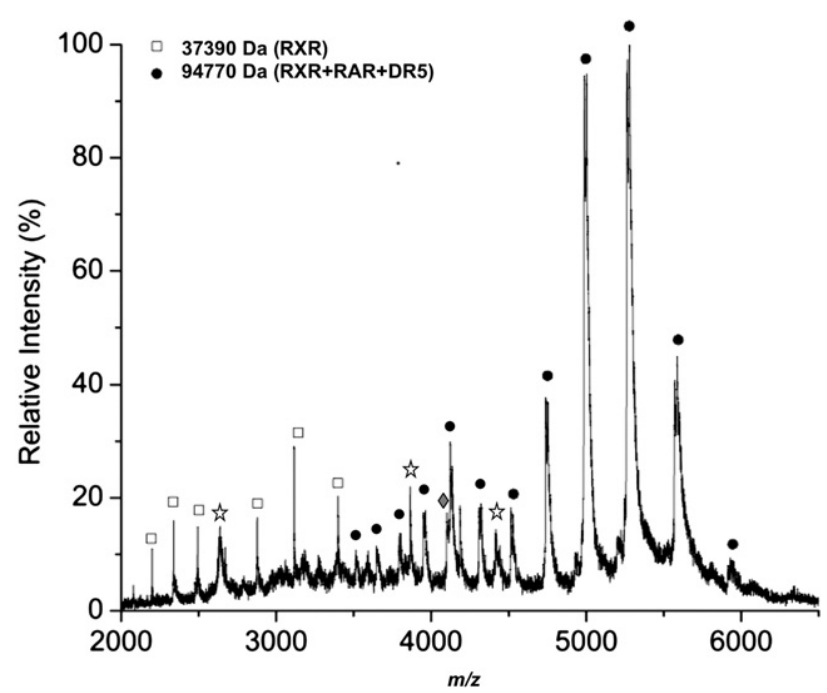

Figure 2. Normalized nanoESI mass spectra of RAR·RXR·dsDR5 in $50 \mathrm{mM} \mathrm{NH} \mathrm{NAc}_{4}$ ( $\mathrm{pH}$ 7.4). [Filled circle] Represents the RA.RAR·RXR·DR5 complex, in good agreement with the theoretical mass $(94,558 \mathrm{Da})$. [Open square] Represents the free RXR left. The grey diamond represents the RAR.RXR.2RA complex and the white stars represent some impurities.

tion process or from dissociation in the gas phase. The sample analyzed here already contained all components (RAR, RXR, RA, and DR5). The individual components were analyzed but not detected by nanoESI due to the high salt content and low protein concentration of the samples, especially in the case of RXR. Desalting was attempted, but did not permit the observation of the compounds.

The formation of the RAR.RXR heterodimer with and without the DNA was also followed by MALDI MS. A spectrum of a mixture of RAR and RXR, in the presence of RA, before cross-linking is shown in Figure 3a. Peaks corresponding to RXR (37.0kDa) and RAR $(44.5 \mathrm{kDa})$ are detected, as well as minor peaks of the doubly charged species of each protein. In the presence of RA, RAR and RXR were then incubated with the K100 cross-linking kit. Following cross-linking, highmass MALDI shows a major signal of the RAR.RXR heterodimer at $84.8 \mathrm{kDa}$, corresponding to the sum of the combined masses of both receptors (Figure $3 b$ ). Both RXR and RAR were also still detected, at $38.3 \mathrm{kDa}$ and $46.4 \mathrm{kDa}$, respectively, as well as the doubly charged complex at $42.4 \mathrm{kDa}$. The observed masses of the proteins and complexes increase by $2 \%-5 \%$ due to the presence of covalently bound cross-linkers. The presence of the free nuclear receptors "decorated" with multiple cross-linker molecules could be a result of an incomplete dimerization. It is also known that the cross-linking process does not always go to completion, thus the presence of free proteins is not surprising. The ligand is not specified on the drawing in the figure, because the MALDI ionization process may destroy the binding between RA and the proteins, which is not stabilized by the cross-linking process due to the struc- 

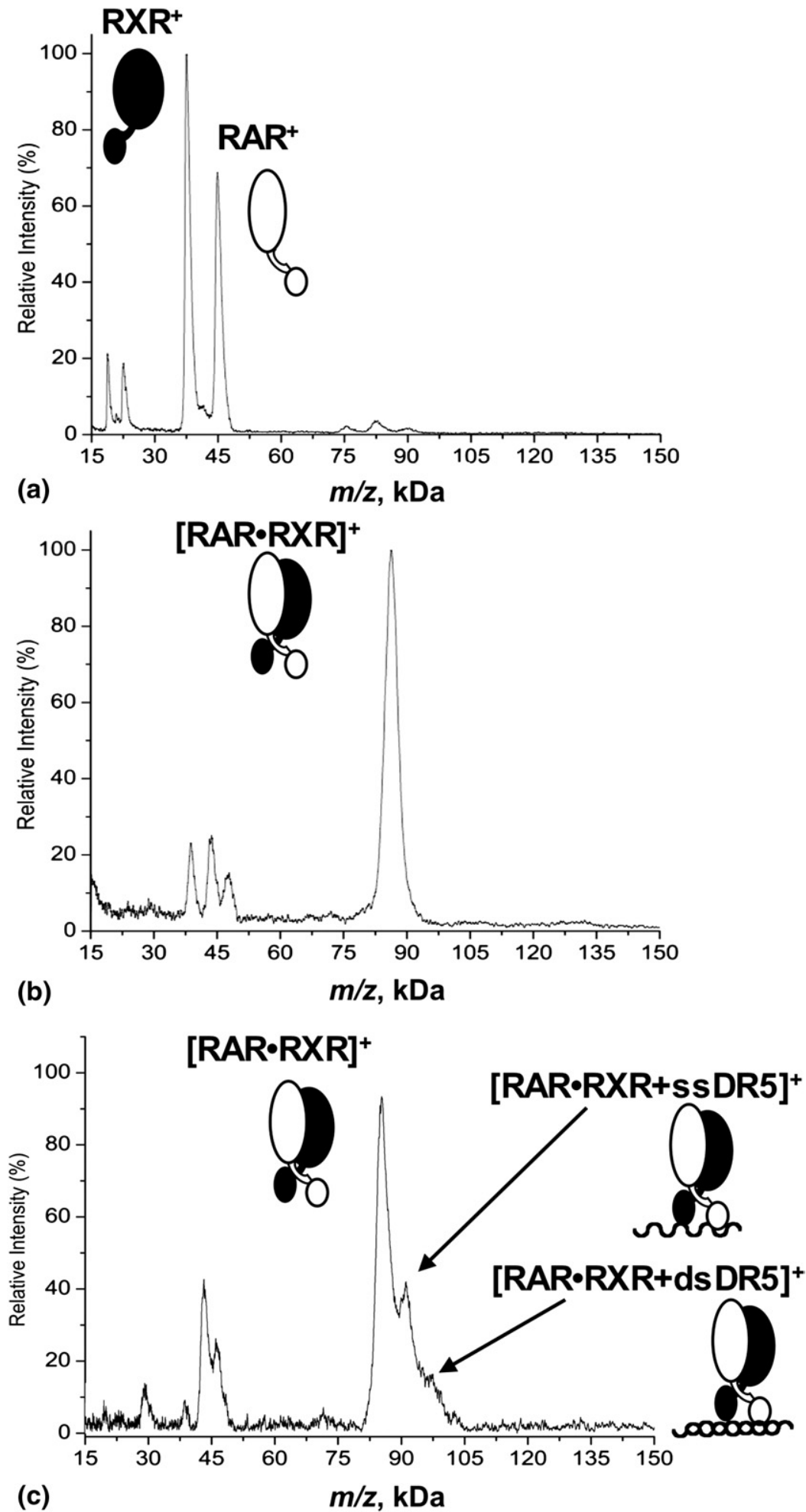

Figure 3. High-mass MALDI mass spectra of the mixture RAR.RXR in the presence of RA. (a) Control spectrum of RAR and RXR proteins without cross-linking. The peaks of the RAR homodimer, the RXR homodimer, and the RAR·RXR heterodimer are observed at a much lower intensity and can be attributed to non-specific dimers, probably induced by clustering, which can occur in the MALDI plume [48, 49]. These gas-phase clusters are distinguishable from specific noncovalent complexes by an exponential intensity decrease and are mainly dependent on the analyte concentration and laser power [50,51]. Lowering the concentration of the analyte or the laser power easily eliminated these unspecific dimers (data not shown). (b) Detection of the RAR.RXR complex after cross-linking. (c) After incubation with DR5, the RAR.RXR complex binds ssDR5 and dsDR5. 
ture of RA. The two receptors were then incubated with DR5 and again submitted to the cross-linking procedure. In Figure 3c, almost all the peaks observed are the same as the ones seen when no DNA is added (Figure 3b). Singly charged RAR and RXR as well as RAR.RXR heterodimer, singly and doubly charged, are detected. One additional peak appears at $90.9 \mathrm{kDa}$, corresponding to the mass of the heterodimer plus a single strand of DR5. In addition, a shoulder at $97.8 \mathrm{kDa}$ also appears, which is attributed to the protein complex bound to the double stranded DR5. These peaks are not present in the control spectrum of the mixture of RA, RAR, RXR and DNA, which was taken before cross-linking (data not shown). As a further control, RAR and RXR, in the presence of RA, were also incubated with DNA samples such as $A_{9}$ and $C_{9}$, which do not contain the specific sequence recognized by the protein complex (AGGTCA). This enabled us to test the specificity of the complex: in all cases, spectra after cross-linking only show the peak corresponding to the proteins or specific protein complex; binding to $A_{9}$ or $C_{9}$ was never observed.

The most probable representation of the mode of interaction between the heterodimer and the DNA is the one shown in Figure 3c. This drawing is based on the known crystal structure of the RAR·RXR·dsDR1 complex and symbolizes the ligand binding domain (large protein lobes) linked to the DNA binding domain (smaller lobes) [11]. It is, however, known that the heterodimer interacts with the DNA as a double strand and not as a single strand. The fact that the peaks corresponding to the RAR.RXR heterodimer plus ssDR5 and dsDR5 are quite weak is most probably due to the low efficiency of the cross-linking reaction with DNA. A further hypothesis to explain the higher abundance of the RAR·RXR·ssDR5 species in the MALDI spectrum would be that during the ionization process, the laser breaks the hydrogen bonds between the two DNA strands complexed to the heterodimer. Possibly the cross-linking produces different species, some with only one strand covalently bound to the protein and others where both strands are covalently bound to the complex. The MALDI ionization process may in the former case disrupt the DNA double strand. Even with multiple hydrogen bonds present in the structure of a dsDNA, special conditions are required to study duplexes with MALDI MS. In the case of protein-DNA, sample conditions (acidic matrix) and laser can be at the origin of the disruption of the dsDNA. Moreover, one study on dsDNA 16-mers reported that to observe the double stranded form by MALDI MS, DNA should have a GC content of more than $75 \%$ [42]. DR5 contains only $50 \%$ of GC in the sequence, which should thus not allow the observation of the duplex. Moreover, MALDI spectra of the dsDR5 alone showed the peak of the single strand to be by far the most abundant species; the dsDR5 was present with much lower intensity (data not shown).

With MALDI MS, the heterodimer and its complexes with ssDR5 and with dsDR5 were detected. In the known crystal structure of the complex consisting of RAR, RXR, and DR1, the receptors interact together while interacting with the DNA [11]. The spatial arrangement in the RAR.RXR.dsDR5 complex is probably quite similar. An observation is that the cross-linking reaction seems to work even in the case of protein-DNA binding, although with lower efficiency compared with the case of protein-protein interactions.

Both nanoESI and MALDI data thus confirm the binding of the heterodimer RAR.RXR with dsDR5. The nanoESI data reflect the solution composition better, i.e., the amount of heterodimer bound to the dsDR5. Very similar information on the interaction can be obtained using cross-linking combined with high-mass MALDI MS.

\section{Mass Spectrometric Characterization of RAR and Effect of DR5 on RAR Dimerization}

Under denaturing conditions, a broad distribution of charge states $(17+$ to $57+)$ that can be assigned to ions of the unfolded RAR monomer was observed by nanoESI (data not shown). Using nondenaturing conditions, the resulting nanoESI spectrum shows three charge state distributions $(\mathrm{m} / \mathrm{z}$ ranges $1000-1600,1600-$ 2250, and 2250-3750), which were assigned to the RAR monomer (data not shown). The deconvoluted molecular weight of $44.59 \mathrm{kDa}$ measured with nanoESI agreed well with the RAR molecular weight based on the known amino acid sequence ( $\mathrm{MW}=44,589 \mathrm{Da})$. The distinct charge state distributions measured under nearnative conditions suggest the coexistence of different RAR conformations in solution. The lowest charge of $\operatorname{RAR}$ ions $(m / z$ range $2250-3750, z=12+, \ldots, 16+)$ is thought to correspond to the most folded RAR conformation (Figure 4a). We then incubated RA and RAR with dsDR5, which contained the specific binding sequence against RAR in a 1:1 ratio. The nanoESI mass spectrum shows a new ion distribution corresponding to a deconvoluted molecular mass of $57.44 \mathrm{kDa}$ (Figure $4 b)$. Compared with the nanoESI mass spectrum of RAR (Figure 4a), this is an increase of $12.85 \mathrm{kDa}$, in excellent agreement with the molecular weight of the dsDR5 measured with nanoESI under nondenaturing conditions $(\mathrm{MW}=12.85 \mathrm{kDa}$, data not shown). The same experiment was performed with an excess of DR5, but in that case, it was only possible to detect the DNA. The conditions used here, optimized to detect the proteinDNA complex, do not reveal the presence of the RA ligand, which probably dissociates in the gas-phase. No homodimer bound to dsDNA was detected with nanoESI, because the expected high $\mathrm{m} / \mathrm{z}$ of this $(101.76$ $\mathrm{kDa}$ ) complex likely is beyond the accessible mass range of our Q-TOF instrument (cf experimental part). Detection of the heterodimer RAR.RXR bound to the dsDNA was possible (cf. Figure 2) albeit likely close to the transmission limits of the instrument. Since the homodimer bound to the DNA is about $7 \mathrm{kDa}$ heavier, 


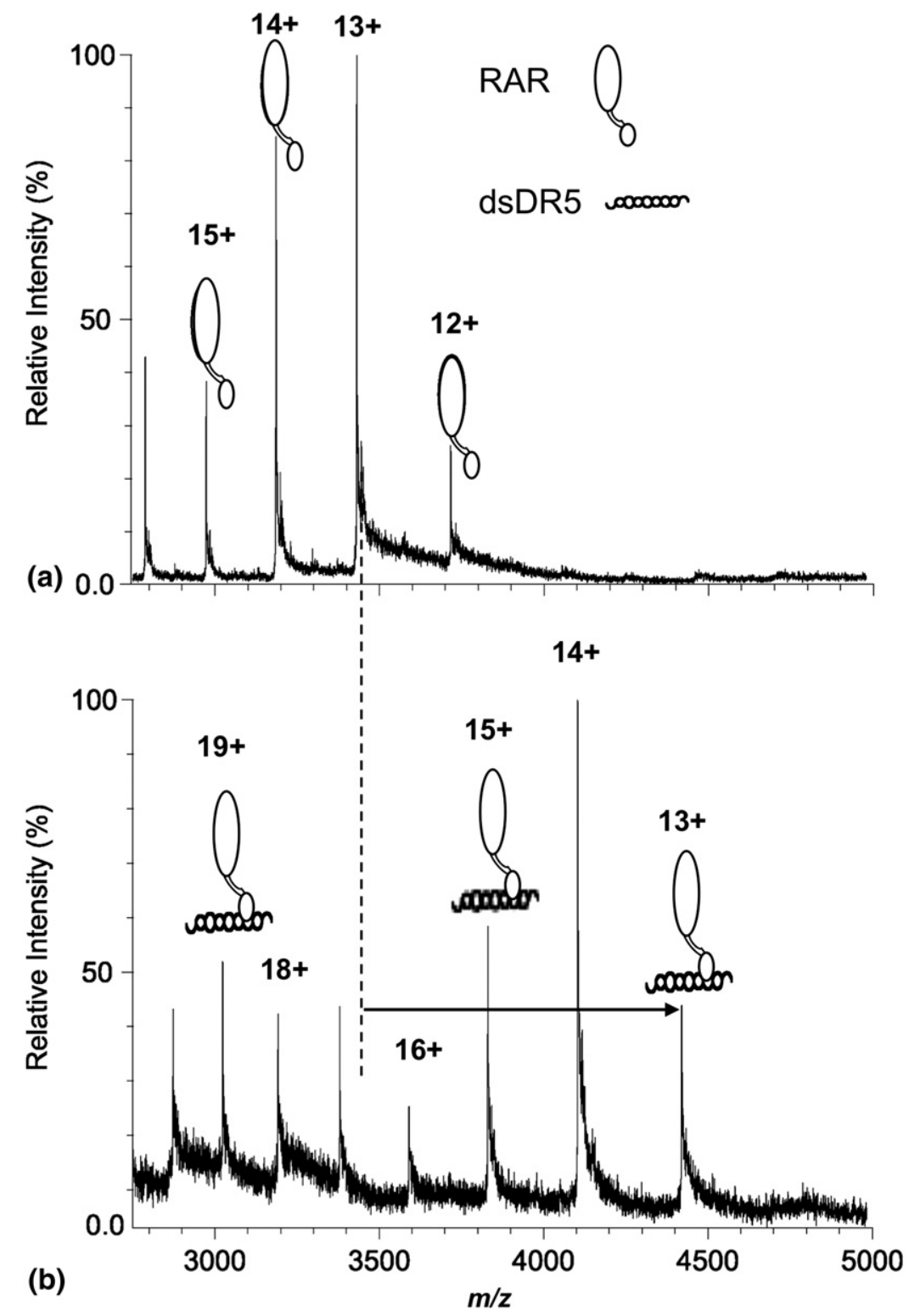

Figure 4. NanoESI mass spectra of RAR with and without DR5, in the presence of RA. Normalized nanoESI mass spectra of (a) RAR in $50 \mathrm{mM} \mathrm{NH}_{4} \mathrm{OAc}(\mathrm{pH} 7.4)$ and (b) RAR incubated with DR5 in 50 $\mathrm{mM} \mathrm{NH}_{4} \mathrm{OAc}$ ( $\mathrm{pH}$ 7.4). Once incubated with DR5, the molecular mass of RAR increased of 12,855 Da, in excellent agreement with the measured molecular weight of the ds DR5.

it is probably just enough to prevent its detection. It would be very interesting to repeat these experiments using a Q-TOF instrument optimized for high $\mathrm{m} / \mathrm{z}$ transmission [40]. Using the exact same conditions as in Figure 2, mainly the dsDR5 signal was observed (data not shown). It was necessary to adapt the ionization and detection conditions for each case, RAR + RXR versus RAR alone, to detect complexes. This is the reason for the broadened peaks in the heterodimer case (Figure 2) compared with the RAR monomer (Figure 4).

After cross-linking and in the presence of RA, highmass MALDI showed essentially RAR monomer (Figure 5a). The peak corresponding to the doubly charged RAR is also present. A small amount of homodimer ions was detected, which can again be attributed to the formation of non-specific RAR clusters during the MALDI ionization process. A control spectrum of RAR without cross-linker shows the same peak with similar intensity (data not shown). After incubating the receptor RAR with RA, an excess of DR5 and with the cross-linkers, high-mass MALDI MS shows a substantial increase of the homodimer intensity at $95.1 \mathrm{kDa}$ (Figure 5B). The measured increase in homodimer abundance suggests a DR5 regulation of the RAR dimerization in solution. Another species is detected, at $\mathrm{m} / \mathrm{z} 53,700$, compatible with a monomer plus a single strand of DR5. Two additional peaks were observed next to the RAR homodimer signal after incubation 


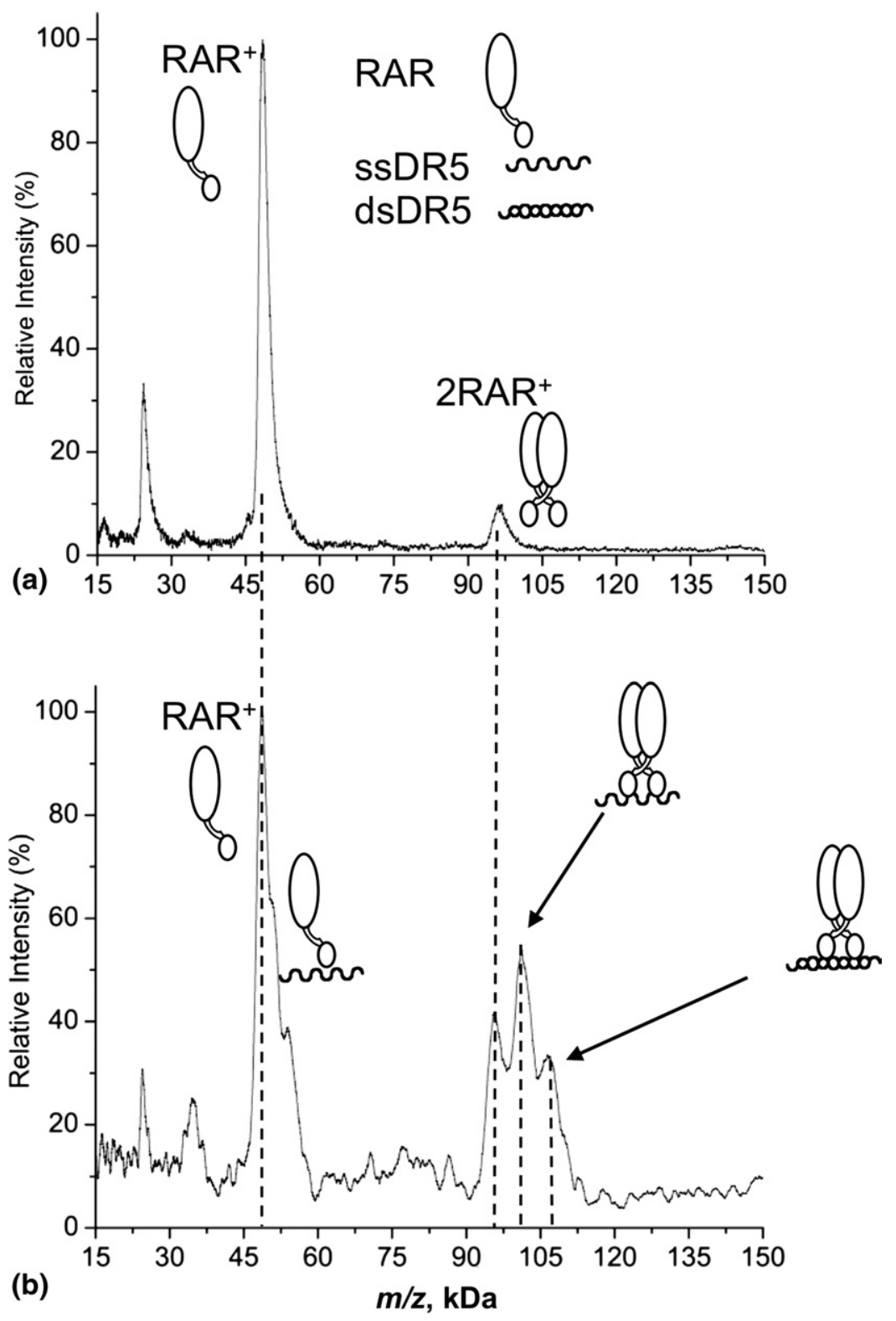

Figure 5. High-mass MALDI mass spectra of RAR without and with DR5, in the presence of RA. Normalized MALDI spectra of RAR (a) after cross-linking stabilization and (b) after cross-linking and incubation with DR5.

with DR5, at 102.3 and $108.7 \mathrm{kDa}$, corresponding to an increase of 7.2 and $13.6 \mathrm{kDa}$ relative to the apo-RAR homodimer (Figure 5b). Considering the low mass accuracy of our MALDI instrument in this mass range and the expected "decoration" due to the cross-linkers, these mass differences are in reasonably good agreement with the molecular weights of the ssDR5 (ssDR5, $\mathrm{MW}=6299$ and $6553 \mathrm{Da})$ and dsDR5 (MW = 12,852 $\mathrm{Da})$. We therefore assign the additional MALDI ion peaks on the RAR homodimer to the bound ssDR5 and dsDR5. The detection by MALDI MS of these two protein-DNA complexes stabilized by cross-linkers supports the notion that the DNA may show some reactivity with the cross-linker. This was investigated separately (see below). Due to the structure of RAR in the RAR·RXR heterodimer complexed with its DNA, the homodimer RAR may interact with DR5 in an analogous fashion as described in the model of Rastinejad et al. [11] The peak at $m / z 108700$ most probably represents the homodimer plus dsDR5 (drawing in Figure $5 b$ ). The higher abundance of the peak corresponding to the homodimer plus single stranded DR5, $m / z 102300$, could be due to partial reaction of the DNA with cross-linker attached on one end to the protein, combined with a disruption of the dsDNA during the ionization process, in analogy to the RAR.RXR case. To verify the binding specificity between DR5 and RAR, the receptor was incubated under similar conditions with single stranded control DNA (i.e., $\mathrm{A}_{9}$ or $\mathrm{C}_{9}$, see Experimental section). No increase in the abundance of 
the homodimer was detected. Furthermore, no complexes were detected (data not shown). The increase of dimer abundance once incubated with DR5 therefore suggests that the homodimerization is induced by the presence of the DNA.

The different intensities for the protein-DNA complexes observed when comparing the RAR.RXR heterodimer and the RAR homodimer can be explained by differences in the sequence of the proteins and in the cross-linking efficiency. The cross-linker reacts preferentially with free amino groups, e.g., side chains of the lysines. Therefore the stabilization should occur more effectively when lysines are abundant on the exterior of a protein. Figure 6 shows the sequence of RAR and RXR, with the DNA binding domain (in black) and the ligand-binding domain (in grey italics). There are more lysines in the sequence of RAR, especially in the DNA binding domain, six compared with only one in the case of RXR (Figure 6). Even in the vicinity of the DNA binding domain (in black in Figure 6), the total number of lysines is a bit larger, 15 for RAR compared with 13 for RXR (in bold). This may provide one explanation for the lower intensity of the RAR.RXR.dsDR5 complex peak compared with the $\mathrm{RAR}_{2} \cdot \mathrm{dsDR} 5$ complex. To investigate a possible ligation of protein and DNA, we studied the reactivity of nucleic acids with NHS esters / cross-linkers in more detail. Incubation of several different single-stranded DNAs with disuccinimidyl suberate (DSS) did not show any reactivity of the DNA. A water-soluble NHS ester containing sulfonate groups, bis(sulfosuccinimidyl) suberate $\left(\mathrm{BS}^{3}\right)$ was also tested. $\left(B^{3}\right)$ did show some reactivity with DNA, albeit an unusual one. Judging from the appearance of a new peak in the MALDI mass spectrum a species that probably resulted from the attack of the sulfonate group on the phosphate of the backbone was created. However, with DSS, which is representative of the components of the K100 crosslinker "cocktail" used here, the DNA clearly showed no reactivity at all.

An alternative explanation for the detection of the protein-DNA complexes by MALDI MS is thus simply ionic interactions between positively charged amino acid residues and the phosphate backbone of DNA. These types of ionic interactions are stable in the gas-phase, and even stronger than in solution [43]. Some studies already described the detection of noncovalent complexes of ss or dsDNA with peptides by MALDI MS [44-47]. This hypothesis is also in line with the fact that RAR contains more $\mathrm{K}, \mathrm{R}$, and $\mathrm{H}$ (23 in total in or around the DNA binding domain, $\mathrm{K}$ in bold, and $\mathrm{R}$ as well as $\mathrm{H}$ underlined in Figure 6) than RXR (15 in total). Lysines and arginines are positively charged at the conditions used for the experiments as well as in the gas-phase. Ionic interactions are thus a likely explanation for the detection of the complexes between the nuclear receptors and DR5 by MALDI MS. We believe that chemical cross-linking enhanced by cooperative effects from the high "local concentration" of crosslinker that has already reacted with amino groups on the protein surface is unlikely.

The different information obtained from the two MS methods originate from the methods themselves. The $m / z$ limitation of our Q-TOF mass spectrometer (i.e., $m / z$ 7000) does not allow the measurement of the native $\mathrm{RAR}_{2} \cdot \mathrm{dsDR} 5(\mathrm{MW}=102 \mathrm{kDa})$. Using native conditions, folded proteins typically generate fairly low charge states that appear in the high mass $m / z$ range, often beyond the limit of the instrumentation. In the case of MALDI MS, the complexes can be destroyed during the ionization process. The monomer with one

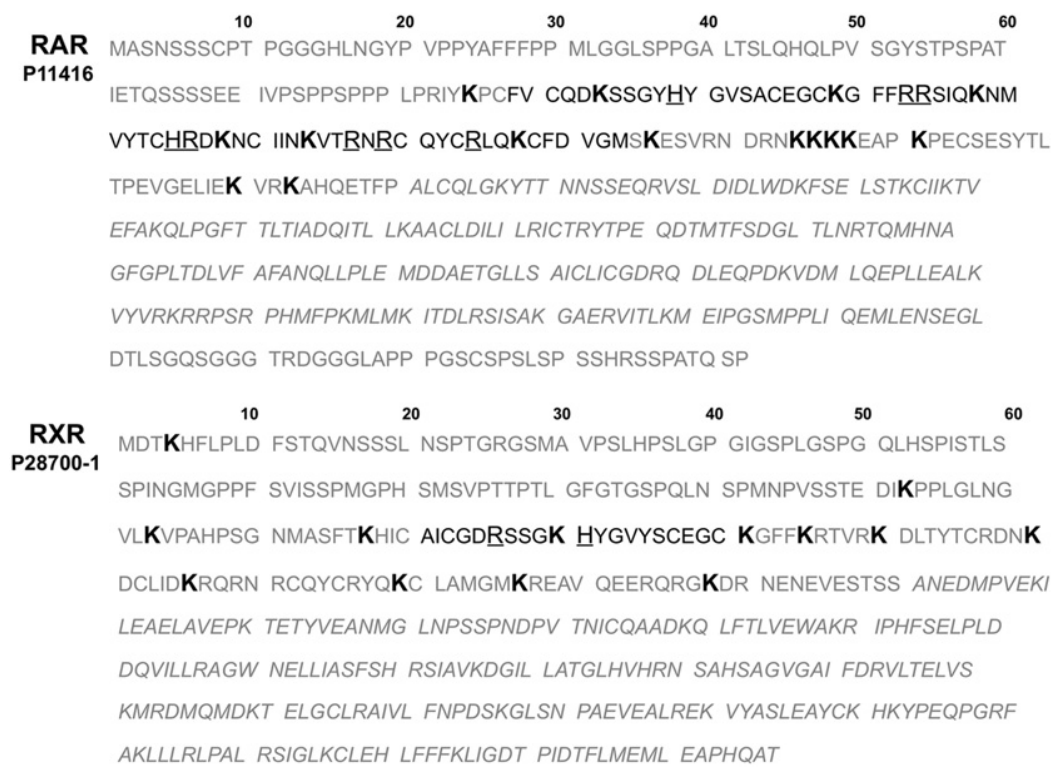

Figure 6. Sequences of RAR and RXR. The DNA binding domain is in black, while the ligand binding domain is in italics. Lysines are highlighted in bold, and arginines as well as histidines, potential positively charged residues, are underlined. 
single strand of DNA bound that appears in the MALDI spectra (Figures $3 c$ and $5 b$ ) is likely due to dissociation of a single DNA strand from the complex, induced by the MALDI process.

\section{Conclusions}

Two complementary mass spectrometric methods were used here to study nuclear receptor protein-DNA complexes. RAR and RXR, in the form of homo- or heterodimers, complexed with DNA were characterized by chemical cross-linking and high-mass MALDI MS as well as by nondenaturing nanoESI MS. In the case of the RAR.RXR heterodimer, nanoESI as well as high-mass MALDI MS showed the complex between RAR.RXR and the dsDR5, although with lower intensity for the latter method. According to the high-mass MALDI MS data, RAR forms a homodimer in solution only when incubated with an excess of DR5. Both ionization methods confirm that dsDR5 interacts with RAR, which shows that complex formation between RAR and the direct repeat motive of the DNA does not require the presence of RXR. The data obtained on these RARRXR/DNA and RAR-RAR/DNA complexes will be helpful for the elucidation of the structure-function relationships of nuclear receptors and for the development of efficient therapies in RAR-mediated diseases.

\section{Acknowledgments}

The authors acknowledge support for this work by a grant from the Swiss National Science Foundation (200020-111831) and by CNRS, INSERM, Université de Strasbourg, the European Commission Structural Proteomics in Europe SPINE2-Complexes (LSHGCT-2006-031220) under the integrated program, Quality of Life and Management of Living Resources. The authors thank David Touboul, Fabio DeGiacomo, and Nicolas Bogliotti (ETH Zurich) for helpful scientific discussions, as well as Laila Hossain and Matthias C. Jecklin for help with the English language.

\section{References}

1. Mascrez, B.; Ghyselinck, N. B.; Chambon, P.; Mark, M. A Transcriptionally Silent RXR $\alpha$ Supports Early Embryonic Morphogenesis and Heart Development. Natl. Acad. Sci. U.S.A. 2009, 106, 4272-4277.

2. Zhang, X. K.; Lehmann, J.; Hoffmann, B.; Dawson, M. I.; Cameron, J.; Graupner, G.; Hermann, T.; Tran, P.; Pfahl, M. Homodimer Formation of Retinoid X-Receptor Induced by 9-cis-Retinoic Acid. Nature 1992, 358, 587-591.

3. Mangelsdorf, D. J.; Evans, R. M. The RXR Heterodimers and Orphan Receptors. Cell 1995, 83, 841-850.

4. Benoit, G. R.; Flexor, M.; Besancon, F.; Altucci, L.; Rossin, A.; Hillion, J.; Balajthy, Z.; Legres, L.; Segal-Bendirdjian, E.; Gronemeyer, H.; Lanotte, M. Autonomous Rexinoid Death Signaling is Suppressed by Converging Signaling Pathways in Immature Leukemia Cells. Mol. Endocrinol. 2001, 15, 1154-1169.

5. Mic, F. A.; Molotkov, A.; Benbrook, D. M.; Duester, G. Retinoid Activation of Retinoic Acid Receptor but Not Retinoid X Receptor is Sufficient to Rescue Lethal Defect in Retinoic Acid Synthesis. Natl. Acad. Sci. U.S.A. 2003, 100, 7135-7140.

6. de Lera, A. R.; Bourguet, W.; Altucci, L.; Gronemeyer, H. Design of Selective Nuclear Receptor Modulators: RAR and RXR as a Case Study. Nat. Rev. Drug Discov. 2007, 6, 811-820.

7. Leid, M.; Kastner, P.; Lyons, R.; Nakshatri, H.; Saunders, M.; Zacharewski, T.; Chen, J. Y.; Staub, A.; Garnier, J. M.; Mader, S.; Chambon, P. Purification, Cloning, and RXR Identity of the HeLa-Cell Factor with Which RAR or TR Heterodimerizes to Bind Target Sequences Efficiently. Cell 1992, 68, 377-395.
8. Germain, P.; Chambon, P.; Eichele, G.; Evans, R. M.; Lazar, M. A.; Leid, M.; De Lera, A. R.; Lotan, R.; Mangelsdorf, D. J.; Gronemeyer, H. International Union of Pharmacology LX Retinoic Acid Receptors. Pharmacol. Rev. 2006, 58, 712-725.

9. Chambon, P. A Decade of Molecular Biology of Retinoic Acid Receptors. FASEB J. 1996, 10, 940-954.

10. Mangelsdorf, D. J.; Umesono, K.; Kliewer, S. A.; Borgmeyer, U.; Ong, E. S.; Evans, R. M. A Direct Repeat in the Cellular Retinol-Binding Protein Type-Ii Gene Confers Differential Regulation by RXR and RAR. Cell 1991, 66, 555-561.

11. Rastinejad, F.; Wagner, T.; Zhao, Q.; Khorasanizadeh, S. Structure of the RXR-RAR DNA-Binding Complex on the Retinoic Acid Response Element DR1. EMBO J. 2000, 19, 1045-1054.

12. Zhao, Q.; Chasse, S. A.; Devarakonda, S.; Sierk, M. L.; Ahvazi, B.; Rastinejad, F. Structural Basis of RXR-DNA Interactions. J. Mol. Biol. 2000, 296, 509-520.

13. Rochel, N.; Ciesielski, F.; Godet, J.; Moman, E.; Roessle, M.; Peluso-Iltis, C.; Mely, Y.; Svergun, D.; Moras, D. Common Architecture of Nuclear Receptor Heterodimers on DNA Direct Repeats, (submitted 2009) unpublished.

14. Vernet, N.; Dennefeld, C.; Guillou, F.; Chambon, P.; Ghyselinck, N. B.; Mark, M. Prepubertal Testis Development Relies on Retinoic Acid but Not Rexinoid Receptors in Sertoli Cells. EMBO J. 2006, 25, 5816-5825.

15. Fenn, J. B.; Mann, M.; Meng, C. K.; Wong, S. F.; Whitehouse, C. M. Electrospray Ionization for Mass-Spectrometry of Large Biomolecules. Science 1989, 246, 64-71.

16. Karas, M.; Hillenkamp, F. Laser Desorption Ionization of Proteins with Molecular Masses Exceeding 10,000 Daltons. Anal. Chem. 1988, 60, 2299-2301.

17. Bolbach, G. Matrix-Assisted Laser Desorption/Ionization Analysis of Noncovalent Complexes: Fundamentals and Applications. Curr. Pharmaceut. Design 2005, 11, 2535-2557.

18. Loo, J. A. Studying Noncovalent Protein Complexes by Electrospray Ionization Mass Spectrometry. Mass Spectrom. Rev. 1997, 16, 1-23.

19. Heck, A. J. R. Native Mass Spectrometry: A Bridge Between Interactomics and Structural Biology. Nat. Methods 2008, 5, 927-933.

20. Schleuder, D.; Hillenkamp, F.; Strupat, K. IR-MALDI-Mass Analysis of Electroblotted Proteins Directly from the Membrane: Comparison of Different Membranes, Application to On-Membrane Digestion and Protein Identification by Database Searching. Anal. Chem. 1999, 71, 3238-3247.

21. Dreisewerd, K.; Rohlfing, A.; Spottke, B.; Urbanke, C.; Henkel, W. Characterization of Whole Fibril-Forming Collagen Proteins of Types I, III, and $\mathrm{V}$ from Fetal Calf Skin by Infrared Matrix-Assisted Laser Desorption Ionization Mass Spectrometry. Anal. Chem. 2004, 76, 3482-3491.

22. Gross, J.; Strupat, K. Matrix-Assisted Laser Desorption/Ionization Mass Spectrometry Applied to Biological Macromolecules. Trac-Trends Anal. Chem. 1998, 17, 470-484

23. Strupat, K.; Sagi, D.; Bonisch, H.; Schafer, G.; Peter-Katalinic, J. Oligomerization and Substrate Binding Studies of the Adenylate Kinase from Sulfolobus acidocaldarius by Matrix-Assisted Laser Desorption/ Ionization Mass Spectrometry. Analyst 2000, 125, 563-567.

24. Friess, S. D.; Daniel, J. M.; Zenobi, R. Probing the Surface Accessibility of Proteins with Noncovalent Receptors and MALDI Mass Spectrometry. Phys. Chem. Chem. Phys. 2004, 6, 2664-2675.

25. Nazabal, A.; Wenzel, R. J.; Zenobi, R. Immunoassays with Direct Mass Spectrometric Detection. Anal. Chem. 2006, 78, 3562-3570.

26. Bich, C.; Scott, M.; Panagiotidis, A.; Wenzel, R. J.; Nazabal, A.; Zenobi, R. Characterization of Antibody-Antigen Interactions: Comparison Between Surface Plasmon Resonance Measurements and High-Mass Matrix-Assisted Laser Desorption/Ionization Mass Spectrometry. Anal. Biochem. 2008, 375, 35-45.

27. Riek, U.; Scholz, R.; Konarev, P.; Rufer, A.; Suter, M.; Nazabal, A.; Ringler, P.; Chami, M.; Muller, S. A.; Neumann, D.; Forstner, M.; Hennig, M.; Zenobi, R.; Engel, A.; Svergun, D.; Schlattner, U.; Wallimann, T. Structural Properties of AMP-Activated Protein KinaseDimerization, Molecular Shape, and Changes Upon Ligand Binding. J. Biol. Chem. 2008, 283, 18331-18343.

28. Akashi, S.; Nagakura, S.; Yamamoto, S.; Okuda, M.; Ohkuma, Y.; Nishimura, Y. Structural Characterization of Human General Transcription Factor TFIIF in Solution. Protein Sci. 2008, 17, 389-400.

29. Farmer, T. B.; Caprioli, R. M. Determination of Protein-Protein Interactions by Matrix-Assisted Laser Desorption/Ionization Mass Spectrometry. J. Mass Spectrom. 1998, 33, 697-704.

30. Sinz, A. Chemical Cross-Linking and Mass Spectrometry for Mapping Three-Dimensional Structures of Proteins and Protein Complexes. I. Mass Spectrom. 2003, 38, 1225-1237.

31. Sinz, A. Chemical Cross-Linking and Mass Spectrometry to Map Three-Dimensional Protein Structures and Protein-Protein Interactions. Mass Spectrom. Rev. 2006, 25, 663-682.

32. Mansuy-Schlick, V.; Delage-Mourroux, R.; Jouvenot, M.; Boireau, W. Strategy of Macromolecular Grafting onto a Gold Substrate Dedicated to Protein-Protein Interaction Measurements. Biosens. Bioelectron. 2006, 21, 1830-1837.

33. Zhang, L.; Rayner, S.; Katoku-Kikyo, N.; Romanova, L.; Kikyo, N. Successful Coimmunoprecipitation of Oct4 and Nanog Using CrossLinking. Biochem. Biophys. Res. Commun. 2007, 361, 611-614.

34. Jensen, O. N.; Barofsky, D. F.; Young, M. C.; Vonhippel, P. H. Swenson, S.; Seifried, S. E. Direct Observation of UV-Cross-Linked Protein Nucleic-Acid Complexes by Matrix-Assisted Laser-Desorption 
Ionization Mass-Spectrometry. Rapid Commun. Mass Spectrom. 1993, 7, 496-501.

35. Ho, D. T.; Sauve, D. M.; Roberge, M. Detection and Isolation of DNA-Binding Proteins Using Single-Pulse Ultraviolet-Laser CrossLinking. Anal. Biochem. 1994, 218, 248-254.

36. Kuhn-Holsken, E.; Dybkov, O.; Sander, B.; Luhrmann, R.; Urlaub, H. Improved Identification of Enriched Peptide-RNA Cross-Links from Ribonucleoprotein Particles (RNPs) by Mass Spectrometry. Nucleic Acids Res. 2007, 35, e95.

37. Klaholz, B. P.; Renaud, J. P.; Mitschler, A.; Zusi, C.; Chambon, P.; Gronemeyer, H.; Moras, D. Conformational Adaptation of Agonists to the Human Nuclear Receptor RAR $\gamma$. Nat. Struct. Biol. 1998, 5, 199-202.

38. Bovet, C.; Wortmann, A.; Eiler, S.; Granger, F.; Ruff, M.; Gerrits, B.; Moras, D.; Zenobi, R. Estrogen Receptor-Ligand Complexes Measured by Chip-Based Nanoelectrospray Mass Spectrometry: An Approach for the Screening of Endocrine Disruptors. Protein Sci. 2007, 16, $938-946$.

39. Ashcroft, A. E. Recent Developments in Electrospray Ionization Mass Spectrometry: Noncovalently Bound Protein Complexes. Nat. Prod. Reports 2005, 22, 452-464.

40. Sobott, F.; Hernandez, H.; McCammon, M. G.; Tito, M. A.; Robinson, C. V. A Tandem Mass Spectrometer for Improved Transmission and Analysis of Large Macromolecular Assemblies. Anal. Chem. 2002, 74, 1402-1407.

41. Karas, M.; Bahr, U.; Hillenkamp, F. Uv Laser Matrix Desorption Ionization Mass-Spectrometry of Proteins in the 100,000 Dalton Range. Int. J. Mass Spectrom. Ion Processes 1989, 92, 231-242.

42. Sudha, R.; Zenobi, R. The Detection and Stability of DNA Duplexes Probed by MALDI Mass Spectrometry. Helv. Chim. Acta 2002, 85, 3136-3143.

43. Daniel, J. M.; Friess, S. D.; Rajagopalan, S.; Wendt, S.; Zenobi, R. Quantitative Determination of Noncovalent Binding Interactions Using Soft Ionization Mass Spectrometry. Int. J. Mass Spectrom. 2002, 216, 1-27.
44. Lin, S. H.; Cotter, R. J.; and Woods, A. S. Detection of Noncovalent Interaction of Single- and Double-Stranded DNA with Peptides by MALDI-TOF. Proteins: Struct. Funct. Genet. 1998, 12-21.

45. Tang, X. D.; Callahan, J. H.; Zhou, P.; Vertes, A. Noncovalen Protein-Oligonucleotide Interactions Monitored by Matrix-Assisted Laser Desorption/Ionization Mass-Spectrometry. Anal. Chem. 1995, $67,4542-4548$.

46. Terrier, P.; Tortajada, J.; Zin, G.; Buchmann, W. Noncovalent Complexes Between DNA and Basic Polypeptides or Polyamines by MALDI-TOF. J. Am. Soc. Mass Spectrom. 2007, 18, 1977-1989.

47. Cheng, X. H.; Morin, P. E.; Harms, A. C. Bruce, J. E.; Ben-David, Y Smith, R. D. Mass Spectrometric Characterization of Sequence-Specific Complexes of DNA and Transcription Factor PU 1 DNA Binding Fomain. Anal. Biochem. 1996, 239, 35-40.

48. Karas, M.; Gluckmann, M.; Schafer, J. Ionization in Matrix-Assisted Laser Desorption/Ionization: Singly Charged Molecular Ions are the Lucky Survivors. J. Mass Spectrom. 2000, 35, 1-12.

49. Livadaris, V.; Blais, J. C.; Tabet, J. C. Formation of Nonspecific Protein Cluster Ions in Matrix-Assisted Laser Desorption/Ionization: Abundances and Dynamical Aspects. Eur. J. Mass Spectrom. 2000, 6, $409-413$.

50. Cohen, L. R. H.; Strupat, K.; Hillenkamp, F. Analysis of Quaternary Protein Ensembles by Matrix Assisted Laser Desorption/Ionization Mass Spectrometry. J. Am. Soc. Mass Spectrom. 1997, 8, 1046-1052.

51. Perera, I. K.; Allwood, D.; Dyer, P. E.; Oldershaw, G. A. Observation of Mixed Molecular Cluster Ions in Matrix-Assisted UV Laser-Desorption Ionization of High-Mass Protein Mixtures. Int. J. Mass Spectrom. Ion Processes 1995, 145, L9-L16. 